$02.3 ; 13.3$

\title{
Сглаживание тонких поликристаллических пленок AIN кластерными ионами аргона
}

\author{
(С) И.В. Николаев ${ }^{1}$, Н.Г. Коробейщиков ${ }^{1}$, М.А. Роенко ${ }^{1}$, П.В. Гейдт ${ }^{1}$, В.И. Струнин ${ }^{2,3}$ \\ ${ }^{1}$ Новосибирский государственный университет, Новосибирск, Россия \\ ${ }^{2}$ Омский государственный университет им. Ф.М. Достоевского, Омск, Россия \\ ${ }^{3}$ Институт радиофизики и фризической электроники ОНЦ СО РАН, Омск, Россия \\ E-mail: i.nikolaev@nsu.ru
}

Поступило в Редакцию 1 сентября 2020 г.

В окончательной редакции 28 сентября 2020 r.

Принято к публикации 11 декабря 2020г.

Исследована модификация поверхности тонких поликристаллических пленок нитрида алюминия с помощью бомбардировки ионно-кластерным пучком. Обработка проводилась высоко- (105 eV/atom) и низкоэнергетическими $(10 \mathrm{eV} /$ atom) кластерными ионами аргона. C помощью метода атомно-силовой микроскопии с применением спектральной функции шероховатости продемонстрировано высокоэффективное сглаживание наноструктурированной поверхности в широком диапазоне пространственных частот $\left(v=0.02-128 \mu \mathrm{m}^{-1}\right)$ при ультрамалой глубине травления $(<100 \mathrm{~nm})$.

Ключевые слова: ионно-кластерный пучок, тонкие пленки, нитрид алюминия, сглаживание поверхности.

DOI: 10.21883/PJTF.2021.06.50759.18536

Обработка материалов слабосвязанными кластерными ионами, генерируемыми ионизацией нейтральных кластеров из сверхзвуковой газовой струи, является многообещающим инструментом для различных практических приложений: приповерхностной имплантации, осаждения тонких пленок, масс-спектрометрии вторичных ионов с помощью кластерных ионов [1]. В частности, нелинейные эффекты взаимодействия кластеров с обрабатываемой поверхностью обеспечивают эффективное сглаживание поверхности различных материалов (металлов, диэлектриков, монокристаллов и сверхтвердых материалов) до субнанометрового уровня шероховатости при минимальном повреждении подповерхностной структуры [2-5].

Нитрид алюминия обладает уникальным набором химических и физических свойств, таких как большие пьезоэлектрические коэффициенты, высокая теплопроводность, высокое напряжение пробоя, хорошая механическая прочность и др. Тонкие пленки на основе нитрида алюминия широко используются в современной микроэлектронике (датчики, силовые полупроводниковые приборы, ультразвуковые преобразователи и т.д.), оптике, оптоэлектронике и других приложениях [6,7]. Поэтому модификация и диагностика материалов (в частности, тонких пленок) на основе AlN представляют собой актуальную задачу для современных приложений. В настоящей работе методом атомно-силовой микроскопии (ACM) с использованием функции спектральной плотности мощности (СПМ, power spectral density, PSD) шероховатости исследовано влияние кластерных ионов аргона на топографию поверхности поликристаллической пленки AlN.
Поликристаллические пленки AlN толщиной $1 \mu \mathrm{m}$ были синтезированы методом магнетронного распыления мишени из чистого $\mathrm{Al}(99.99 \%)$ при постоянной мощности магнетрона $600 \mathrm{~W}$. Формирование пленок AIN осуществлялось на подложке из ситалла СТ-50-1-1-0.6 в азот-аргоновой среде при расходе рабочих газов $5 \mathrm{sccm}$ для $\mathrm{N}_{2}$ и $4 \mathrm{sccm}$ для Ar. Фоновое давление в вакуумной камере составляло $0.07 \mathrm{~Pa}$. Температура подложки поддерживалась на уровне $623 \mathrm{~K}$. Обработка пленок AlN проводилась интенсивным несепарированным по размерам кластеров ионно-кластерным пучком аргона на экспериментальной установке КЛИУС [8]. Средний размер кластеров $N$ варьировался за счет изменения давления торможения в источнике газа и определялся по методике, описанной в [9]. Эксперименты выполнялись при нормальном падении кластерных ионов на поверхность мишени.

Известно, что при кластерной бомбардировке сглаживание поверхности происходит за счет двух параллельных процессов: распыления приповерхностных атомов мишени и их латерального перемещения. Как для наклонных углов [9-11], так и для нормального падения кластеров [9] коэффициент распыления мишени определяется отношением кинетической энергии кластеров $E$ к их среднему размеру $N$. При высоких энергиях $E / N$ преобладает процесс распыления, и значительная часть начальной кинетической энергии $E$ тратится на выбивание атомов мишени [11]. Предполагается, что при уменьшении $E / N$ от 100 до нескольких eV/atom начальная энергия кластеров преимущественно тратится на перемещение атомов мишени в приповерхностном слое и уносится рассеянными атомами кластеров. В результате коэффициент распыления нелинейно уменьшается почти 
Таблица 1. Главные параметры режимов обработки

\begin{tabular}{|c|c|c|c|c|}
\hline $\begin{array}{c}\text { Режим } \\
\text { обработки }\end{array}$ & $\begin{array}{c}\text { Средний размер } \\
\text { кластеров } N \text {, } \\
\text { atoms/cluster }\end{array}$ & $\begin{array}{c}\text { Энергия атома в } \\
\text { кластере } E / N, \\
\text { eV/atom }\end{array}$ & $\begin{array}{c}\text { Плотность тока ионно- } \\
\text { кластерного пучка } j, \\
\mu \mathrm{A} / \mathrm{cm}^{2}\end{array}$ & $\begin{array}{c}\text { Доза } \\
\text { облучения, } \\
\text { ions } / \mathrm{cm}^{2}\end{array}$ \\
\hline $\begin{array}{l}1 \\
2\end{array}$ & $\begin{array}{r}210 \\
1000\end{array}$ & $\begin{array}{r}105 \\
10\end{array}$ & $\begin{array}{l}0.35 \\
0.50\end{array}$ & $\begin{array}{l}8.1 \cdot 10^{15} \\
2.2 \cdot 10^{16}\end{array}$ \\
\hline
\end{tabular}
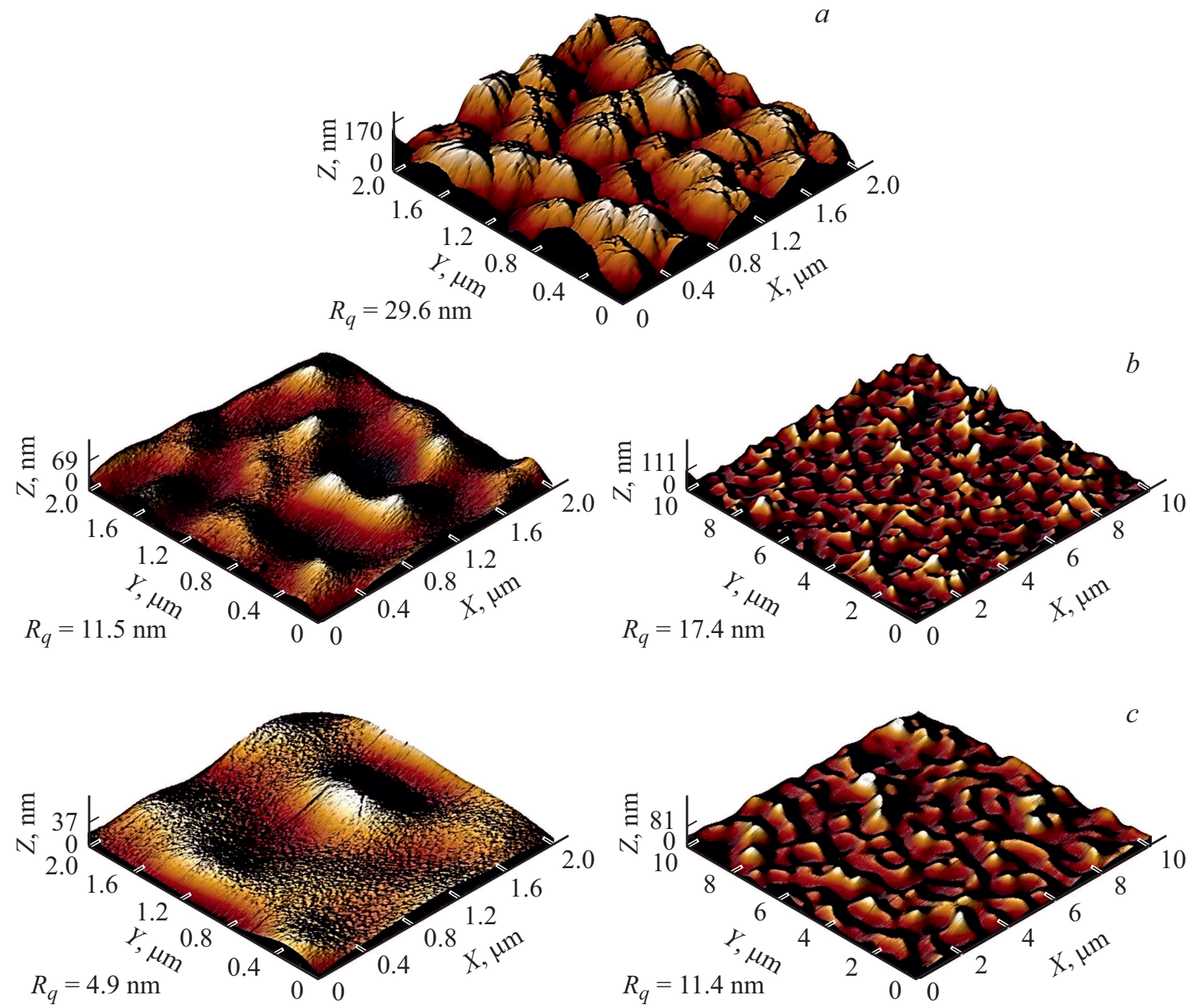

Рис. 1. ACM-изображения поверхности пленки AlN при размерах области сканирования $2 \times 2$ и $10 \times 10 \mu$ m. $a-$ исходная поверхность; $b$ - после обработки в режиме $1 ; c$ - после обработки в режиме 2.

на три порядка (примерно с 1 до 0.001) [9]. Исходя из этого для обработки образцов AlN были выбраны два режима: высоко- (105 eV/atom) и низкоэнергетический (10 eV/atom). Основные параметры режимов обработки приведены в табл. 1 .

Топография поверхности образцов исследовалась методом ACM. Измерения проводились на приборе NTEGRA Prima HD (NT-MDT) в контактном режиме с помощью высокоточных ACM-зондов ETALON HA_C (NT-MDT) с радиусом закругления менее $10 \mathrm{~nm}$ и пространственным разрешением $1024 \times 1024$ пикселей. Для повышения достоверности АСМ-измерения были проведены до и после обработки в одних и тех же областях поверхности мишени, расположенных на расстоянии $3 \mathrm{~mm}$ друг от друга. Глубина травления определялась путем измерения „ступеньки“, формируемой под маской на левой и правой границах обработанной области. На каждой границе были выбраны три области измерения размерами $100 \times 100 \mu \mathrm{m}$. В каждой области были построены три профиля ступеньки. Средние скорости травления поверхности мишени, определяемые отношением усредненной высоты ступеньки и времени обработки, составили 1.5 и $0.25 \mathrm{~nm} / \mathrm{min}$ в режимах 1 и 2 соответственно. 
Таблица 2. Характеристики шероховатости поверхности нитрида алюминия

\begin{tabular}{|c|c|c|c|c|c|c|c|c|c|}
\hline \multirow{3}{*}{ Режим обработки } & \multicolumn{8}{|c|}{ Размер области сканирования, $\mu \mathrm{m}$} & \multirow{3}{*}{$\begin{array}{c}\sigma_{e f f}^{\text {total }}, \\
\mathrm{nm}\end{array}$} \\
\hline & \multicolumn{2}{|c|}{$2 \times 2$} & \multicolumn{2}{|c|}{$10 \times 10$} & \multicolumn{2}{|c|}{$40 \times 40$} & \multicolumn{2}{|c|}{$100 \times 100$} & \\
\hline & $\begin{array}{c}\left\langle R_{q}\right\rangle \\
\mathrm{nm}\end{array}$ & $\begin{array}{c}\left\langle\sigma_{e f f}\right\rangle, \\
\mathrm{nm}\end{array}$ & $\begin{array}{c}\left\langle R_{q}\right\rangle, \\
\mathrm{nm}\end{array}$ & $\begin{array}{c}\left\langle\sigma_{e f f}\right\rangle, \\
\mathrm{nm}\end{array}$ & $\begin{array}{c}\left\langle R_{q}\right\rangle, \\
\mathrm{nm}\end{array}$ & $\begin{array}{c}\left\langle\sigma_{e f f}\right\rangle, \\
\mathrm{nm}\end{array}$ & $\begin{array}{c}\left\langle R_{q}\right\rangle, \\
\mathrm{nm}\end{array}$ & $\begin{array}{c}\left\langle\sigma_{e f f}\right\rangle, \\
\mathrm{nm}\end{array}$ & \\
\hline $\begin{array}{c}\text { Исходная } \\
\text { поверхность }\end{array}$ & 29.2 & 30.0 & 35.9 & 36.4 & 26.4 & 28.1 & 21.3 & 22.3 & 41.0 \\
\hline После режима 1 & 9.9 & 10.0 & 16.8 & 16.9 & 14.3 & 15.1 & 12.3 & 12.6 & 20.6 \\
\hline После режима 2 & 4.9 & 4.8 & 11.3 & 11.1 & 11.1 & 11.7 & 9.5 & 9.9 & 14.0 \\
\hline
\end{tabular}

П р и м е ч а н и е. $\left\langle R_{q}\right\rangle$ - среднеквадратичная шероховатость, $\left\langle\sigma_{e f f}\right\rangle-$ средняя эффективная шероховатость, $\sigma_{e f f}^{\text {total }}-$ эффективная шероховатость, обобщенная по всему измеренному диапазону пространственных частот $v$.

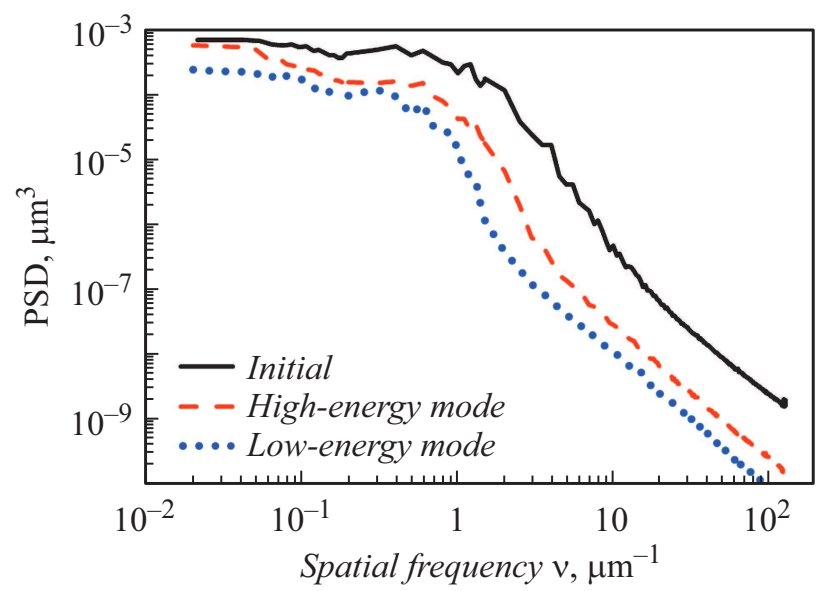

Рис. 2. Функция спектральной плотности мощности шероховатости до и после обработки ионно-кластерным пучком аргона.

На рис. 1 представлены 3D ACM-изображения поверхности пленки AlN до и после обработки ионно-кластерным пучком аргона. Как правило, при магнетронном распылении монокристаллы AlN растут на мелких равноосных зернах и с увеличением толщины пленки приобретают столбчатую структуру $[12,13]$. В наших образцах средний диаметр столбчатых микрокристаллов составлял около $330 \mathrm{~nm}$.

СПМ-функции шероховатости на различных масштабах области сканирования $(2 \times 2,10 \times 10,40 \times 40$ и $100 \times 100 \mu \mathrm{m})$ позволяют корректно охарактеризовать топографию поверхности в широком диапазоне пространственных частот $v$ и определить эффективную шероховатость поверхности $\sigma_{e f f}[8,14]$. Усредненные значения эффективной $\sigma_{e f f}$ и среднеквадратичной $R_{q}$ шероховатости поверхности при различных размерах области сканирования до и после обработки показаны в табл. 2. Профили поверхности на рис. 1 и данные табл. 2 наглядно показывают, что шероховатость поверхности AIN значительно уменьшилась на всех масштабах сканирования после обработки кластерными ионами в обоих режимах. После обработки в низкоэнергетическом режиме на малых масштабах $(2 \times 2 \mu \mathrm{m})$ наблюдается максимальное снижение шероховатости - в 6 раз, а при бо́льших размерах области сканирования $(10 \times 10 \mu \mathrm{m})$ шероховатость снижается в 3.2 раза.

Следует уточнить, что для достоверной характеризации шероховатости, особенно в случае наноструктурированной поверхности, важную роль играет размер области сканирования. Как видно из табл. 2, наибольшая шероховатость наблюдалась на масштабе $10 \times 10 \mu \mathrm{m}$, т. е. при размере пикселя около $10 \times 10 \mathrm{~nm}$. Причинами уменьшения параметров шероховатости при бо́льших масштабах являются отсутствие неровностей больших латеральных размеров и снижение разрешающей способности, а при меньших масштабах $(2 \times 2 \mu \mathrm{m})-$ небольшое количество монокристаллитов для корректного отображения их размеров на данном масштабе. Таким образом, параметры шероховатости на масштабе $10 \times 10 \mu \mathrm{m}$ являются наиболее корректными для анализа наноструктур соответствующих размеров.

На рис. 2 приведены обобщенные СПМ-функции поверхности пленки AIN до и после обработки кластерами. Видно, что в результате обработки шероховатость значительно понизилась во всем измеряемом диапазоне пространственных частот $v=0.02-128 \mu \mathrm{m}^{-1}$. Данный диапазон частот соответствует неровностям с характерными размерами от нескольких нанометров до $50 \mu \mathrm{m}$. Ранее $[5,8]$ нами было показано, что для сверхгладких поверхностей $\left(R_{q} \leqslant 1 \mathrm{~nm}\right)$ различных материалов обработка кластерными ионами обеспечивает сглаживание в одном и том же более узком диапазоне $v=0.2-128 \mu \mathrm{m}^{-1}$.

Важнейшей характеристикой процесса обработки является глубина травления. В нашем случае глубина травления составила всего 90 и $30 \mathrm{~nm}$ для высокои низкоэнергетического режимов соответственно. При этом обобщенная эффективная шероховатость $\sigma_{e f f}^{\text {total }}$, определяемая как интеграл СПМ-функции [9], после обработки кластерами с энергией $E / N=10 \mathrm{eV} /$ atom coставляет $14 \mathrm{~nm}$, что в 1.5 раза меньше соответствующего значения для поверхности, полученной после обработки в режиме 1, и в 2.9 раза ниже, чем $\sigma_{e f f}^{\text {total }}$ для исходной поверхности. 
Таким образом, полученные результаты свидетельствуют о том, что газовые кластерные ионы могут успешно использоваться для высокоэффективного сглаживания поликристаллической поверхности различных материалов. Данным методом обеспечивается сглаживание неровностей с латеральными размерами до $50 \mu \mathrm{m}$ при ультрамалой глубине распыления (десятки нанометров). Наибольшая эффективность достигается в режимах при малой удельной энергии кластеров (единицы eV/atom), что обусловлено активным латеральным перемещением приповерхностных атомов мишени.

\section{Благодарности}

Работа выполнена с использованием оборудования ЦКП „Прикладная физика“ НГУ.

\section{Финансирование работы}

Работа выполнена при финансовой поддержке Минобрнауки РФ (гранты FSUS-2020-0039, FSUS-20200029) и госзадания ОНЦ СО РАН (№ 075-03-2020-614).

\section{Конфликт интересов}

Авторы заявляют, что у них нет конфликта интересов.

\section{Список литературы}

[1] I. Yamada, Materials processing by cluster ion beams. History, theory, and applications (CRC Press, Boca Raton, 2015).

[2] I. Yamada, J. Matsuo, N. Toyoda, T. Aoki, T. Seki, Cur. Opin. Solid State Mater. Sci., 19, 12 (2015). DOI: $10.1016 /$ j.cossms.2014.11.002

[3] A.T. Wu, D.R. Swenson, Z. Insepov, Phys. Rev. Spec. Top., 13, 093504 (2010). DOI: 10.1016/j.nimb.2018.02.019

[4] A.E. Ieshkin, D.S. Kireev, Yu.A. Ermakov, A.S. Trifonov, D.E. Presnov, A.V. Garshev, Yu.V. Anufriev, I.G. Prokhorova, V.A. Krupenin, V.S. Chernysh, Nucl. Instrum. Meth. Phys. Res. B, 421, 27 (2018). DOI: 10.1016/j.nimb.2018.02.019

[5] Н.Г. Коробейщиков, И.В. Николаев, М.А. Роенко, Письма в ЖТФ, 45 (6), 30 (2019). DOI: 10.21883/PJTF.2019.06.47496.17646 [Пер. версия: 10.1134/S1063785019030295].

[6] Y.Q. Fu, J.K. Luo, N.T. Nguyen, A.J. Walton, A.J. Flewitt, X.T. Zu, Y. Li, G. McHale, A. Matthews, E. Iborra, H. Du, W.I. Milne, Prog. Mater. Sci., 89, 31 (2017). DOI: 10.1016/j.pmatsci.2017.04.006

[7] V.S. Kudyakova, R.A. Shishkin, A.A. Elagin, M.V. Baranov, A.R. Beketov, J. Eur. Ceram. Soc., 37, 1143 (2017). DOI: $10.1016 /$ j.jeurceramsoc.2016.11.051

[8] N.G. Korobeishchikov, I.V. Nikolaev, M.A. Roenko, Nucl. Instrum. Meth. Phys. Res. B, 438, 1 (2019). DOI: $10.1016 /$ j.nimb.2018.10.019

[9] N.G. Korobeishchikov, I.V. Nikolaev, M.A. Roenko, V.V. Atuchin, Appl. Phys. A, 124, 833 (2018). DOI: $10.1007 / \mathrm{s} 00339-018-2256-3$

[10] M.P. Seah, J. Phys. Chem. C, 117, 12622 (2013). DOI: $10.1021 / \mathrm{jp} 402684 \mathrm{c}$
[11] R.J. Paruch, Z. Postawa, B.J. Garrison, J. Vac. Sci. Technol. B, 34, 03 H105 (2016). DOI: 10.1116/1.4940153

[12] B.-H. Hwang, C.-S. Chen, H.-Y. Lu, T.-C. Hsu, Mater. Sci. Eng. A, 325, 380 (2002). DOI: 10.1016/S0921-5093(01)01477-0

[13] C. Duquenne, M-P. Besland, P.Y. Tessier, E. Gautron, Y. Scudeller, D. Averty, J. Phys. D: Appl. Phys., 45, 015301 (2011). DOI: $10.1088 / 0022-3727 / 45 / 1 / 015301$

[14] N.I. Chkhalo, A.V. Kirsanov, G.A. Luchinin, O.A. Malshakova, M.S. Mikhailenko, A.I. Pavlikov, A.E. Pestov, M.V. Zorina, Appl. Opt., 57, 6911 (2018). DOI: 10.1364/AO.55.001249 